\title{
Indicadores antropométricos de obesidade abdominal: revisão dos artigos indexados na biblioteca SciELO
}

\author{
Anthropometric indicators of abdominal obesity: review of the \\ papers indexed on SciELO electronic library
}

\author{
Cláudio Barnabé dos Santos Cavalcanti \\ Sineide Cristina Barbosa do Egito Carvalho ${ }^{1}$ \\ Mauro Virgilio Gomes de Barros
}

1 Universidade de Pernambuco. Escola Superior de Educação Física. Grupo de Pesquisa em Estilos de Vida e Saúde. Programa de Pós-graduação em Hebiatria. Recife, PE. Brasil.

Recebido em 10/06/08 Revisado em 21/07/08 Aprovado em 07/11/08
Resumo - Recorreu-se à revisão sistemática de artigos originais publicados no período de 1993 a 2007 e indexados na Scientific Electronic Library Online - SCIELO, a fim de identificar os indicadores antropométricos utilizados na detecção da obesidade abdominal em diferentes subgrupos populacionais e sintetizar os resultados observados quanto à relação entre estes indicadores e comorbidades associadas à obesidade. Na análise dos manuscritos, observou-se: ano de publicação; tamanho/faixa etária da amostra; indicador antropométrico empregado para detecção dos casos de obesidade abdominal; e, resultados principais. Foram localizados 47 artigos que atendiam aos critérios de inclusão, 34 dos quais publicados nos últimos cinco anos. A medida da relação cintura-quadril e da circunferência da cintura foram utilizadas como indicador de obesidade abdominal, respectivamente, em 34 e em 11 dos estudos. Na maioria, a amostra foi constituída por grupos heterogêneos em relação à idade (27), sendo que dez incluíam também adolescentes. Das investigações com amostras mais homogêneas em relação à faixa etária, quatro foram com idosos e três com crianças, mas nenhum com amostra exclusiva de adolescentes. Em 26 manuscritos, o tamanho da amostra foi superior a 500 sujeitos. Apesar de diferenças quanto à magnitude, de uma maneira geral, os estudos evidenciaram que os indicadores antropométricos de obesidade abdominal estão correlacionados à ocorrência de hipertensão, diabetes, dislipidemias e risco coronariano elevado. Resultados desta revisão sugerem que a obesidade abdominal está associada à morbidade por doenças metabólicas, mas há escassez de estudos com crianças e adolescentes, fato que denota a necessidade de intensificação da investigação na área.

Palavras-chave: Antropometria; Obesidade; Relação cintura-quadril.

Abstract - A systematic review of original articles published between 1993 and 2007 and indexed in SciELO (Scientific Electronic Library Online) was carried out to identify the anthropometric indicators used to assess abdominal obesity in different target subgroups, and to provide an overview of available evidence regarding the relationship between these anthropometric indicators and obesity-associated comorbidities. The articles were analyzed and the following data were collected: year of publication, sample size and age, anthropometric measure used to detect abdominal obesity, and main results of the study. The review comprised 47 articles which met all inclusion criteria, most of them published over the last 5 years. Waist-hip ratio and waist circumference were used as indicators of abdominal obesity in 34 and 11 of the articles reviewed, respectively. With respect to age range, the samples were heterogeneous in most of the studies $(n=27)$ and ten included adolescents. Four studies were conducted on elderly subjects and three included children, but none exclusively involved adolescents as the target population. The sample size was larger than 500 in 26 studies. Despite disagreement in terms of the degree of correlation, the studies generally showed that the anthropometric indicators of abdominal obesity were correlated with hypertension, diabetes, dyslipidemias, and coronary heart risk. The results of this review suggest that abdominal obesity is associated with metabolic diseases. However, there is a lack of studies involving the adolescent population, suggesting the need for further research in this area.

Key words: Anthropometry; Obesity; Waist-hip ratio. 


\section{INTRODUÇÃO}

A obesidade está sendo considerada uma epidemia mundial, presente tanto em países desenvolvidos como em desenvolvimento'. É uma doença considerada como um dos maiores problemas de saúde da atualidade, devido às comorbidades (hipertensão, diabetes e dislipidemias) associadas à mesma. Trata-se de um agravo que também está associado à progressão da aterosclerose, além de ser causa da ocorrência da chamada "síndrome metabólica".

Segundo a Organização Mundial de Saúde $(\mathrm{OMS})^{3}$, a ocorrência da obesidade reflete a interação entre fatores ambientais e a predisposição genética. Contudo, as evidências quanto a uma maior susceptibilidade de algumas populações em decorrência de fatores genéticos são inconsistentes. Isto sugere que as variáveis alimentares e a atividade física são os fatores responsáveis pelas diferenças quanto à prevalência da obesidade em diferentes grupos populacionais.

A classificação dos casos de obesidade em estudos na área de saúde é efetuada mediante análise da proporção de gordura corporal ou, alternativamente, em estudos populacionais, mediante interpretação de variáveis antropométricas. No caso dos indicadores antropométricos, estes podem ser utilizados para identificação tanto dos casos de obesidade generalizada quanto de obesidade centralizada (andróide), cuja ocorrência é mais freqüente entre os homens. A obesidade centralizada é um fator de risco cardiovascular importante, além de ter maior interferência na homeostase glicose-insulina do que a obesidade generalizada ${ }^{4}$. Há evidências também de que indicadores antropométricos de obesidade abdominal são capazes de predizer a mortalidade por todas as causas e a mortalidade por doenças cardiovasculares ${ }^{5}$.

$\mathrm{Na}$ análise da distribuição de gordura corporal e na detecção da obesidade abdominal, mediante utilização do método antropométrico, diferentes indicadores vem sendo utilizados: relação cinturaquadril (RCQ), circunferência da cintura (CC), índice de conicidade (IC) e relação cintura-estatura (RCEst). A RCQ é obtida pela razão entre os perímetros da cintura e do quadril, o IC é determinado por uma relação matemática entre a massa e a estatura corporal do sujeito e a RCEst pela razão entre o perímetro da cintura e a medida da estatura. Estudos internacionais têm procurado comparar a utilização desses indicadores como critério para triagem de comorbidades associadas à própria obesidade $^{6}$ e como critério para avaliação indireta do risco de doenças cardiovasculares e síndrome metabólica ${ }^{5,7,8}$.

O estudo de Molarius e colaboradores ${ }^{9}$ sugere que, quando comparada a RCQ, a medida isolada da CC está mais fortemente associada à quantidade de gordura abdominal e, por conseguinte, à ocorrência de distúrbios metabólicos. Em estudo recente, Pitanga e Lessa ${ }^{10}$ concluíram que o IC e a RCQ são melhores indicadores de risco coronariano que a CC e o índice de massa corporal. Por sua vez, Hsieh e Muto ${ }^{7}$ verificaram que o melhor indicador antropométrico para avaliação de fatores agregados de risco coronariano em não obesos é a relação cintura-estatura.

Considerando a importância destes indicadores para avaliação da freqüência de obesidade abdominal em estudos populacionais e a aparente falta de convergência entre os resultados dos estudos, efetuou-se uma revisão sistemática dos artigos originais sobre obesidade centralizada publicados em periódicos indexados na Scientific Electronic Library Online-SCIELO. Trata-se, portanto, de um estudo que busca preencher uma importante lacuna de conhecimento, ao efetuar uma síntese do corpo de evidências disponíveis para profissionais de saúde brasileiros quanto ao uso de indicadores antropométricos na detecção e análise das comorbidades e fatores associados à obesidade abdominal.

Os principais objetivos foram: (1) identificar os indicadores antropométricos utilizados na detecção da obesidade abdominal em diferentes subgrupos populacionais; e, (2) sintetizar os resultados observados quanto à inter-relação entre estes indicadores e comorbidades associadas à obesidade.

\section{PROCEDIMENTOS METODOLÓGICOS}

Para o desenvolvimento do presente estudo, recorreu-se à revisão sistemática de artigos originais com resultados de pesquisas sobre obesidade centralizada, publicados em periódicos indexados na Scientific Electronic Library Online - SCIELO, no período de 1993 a 2007. A opção por delimitar a busca à SCIELO foi motivada pelo entendimento de que esta biblioteca eletrônica representa, na atualidade, a fonte mais acessível de consulta para profissionais de saúde e, também, parte importante da produção científica nacional qualificada.

Para localização dos artigos, as buscas foram efetuadas através do portal da BIREME (Biblioteca Virtual em Saúde) e foram limitadas às seguintes bases de dados: MEDLINE, LILACS e ADOLEC. Os mecanismos de busca avançada que permitem a 
combinação de termos e aplicação de limites foram utilizados para realização da revisão, utilizando-se para tanto dos seguintes termos/descritores: cintura (waist), circunferência da cintura (waist circumference), relação cintura-quadril (waist-hip ratio), índice $\mathrm{C}$ (index $\mathrm{C}$ ), obesidade abdominal (abdominal obesity), obesidade (obesity). Adicionalmente, os termos Brasil, brasileiro e brasileira também foram utilizados para refinar a busca.

O resultado inicial, nas três bases de dados, resultou num total de 5.552 artigos. Foram incluídos todos os artigos originais de pesquisas realizadas no Brasil, com seres humanos, independentemente da faixa etária, publicados em língua inglesa, espanhola ou portuguesa e que haviam sido publicados em periódicos indexados na SCIELO. Artigos que veiculavam resultados de estudos analíticos, como ensaios e pesquisas bibliográficas não foram incluídos no estudo. Todos os demais, independentemente do delineamento de investigação foram considerados elegíveis. Após triagem e eliminação dos artigos que não atendiam aos critérios de inclusão anteriormente estabelecidos, 47 foram utilizados no desenvolvimento da presente revisão.

$\mathrm{Na}$ análise de cada artigo foram observados os seguintes aspectos: período de publicação; tamanho e faixa etária da amostra; método(s) utilizado(s) na avaliação da distribuição da gordura corporal; e, resultados principais do estudo. Em relação aos resultados dos estudos, especial enfoque foi dado à identificação de evidência da inter-relação entre os indicadores de obesidade abdominal com pressão sanguínea (hipertensão), controle glicêmico (diabetes), lipoproteínas plasmáticas (dislipidemias) e risco coronariano.

O processo de revisão foi conduzido, de maneira independente, por dois pesquisadores. Cada um elaborou um quadro com a síntese das informações extraídas dos manuscritos e, em seguida, os quadros foram comparados a fim de verificar a concordância entre os pares. $\mathrm{Na}$ eventualidade de divergências entre as informações sintetizadas pelos pares, o artigo foi revisado por um terceiro membro da equipe de pesquisa a fim de identificar as incoerências e definir quais as evidências que o manuscrito efetivamente veiculava. Este procedimento foi realizado conjuntamente pelos três pesquisadores e divergências entre os pares ocorreram na análise de apenas cinco dos 47 artigos incluídos na revisão.

\section{DISCUSSÃO DAS EVIDÊNCIAS}

Mediante aplicação das estratégias de busca, identificou-se 47 manuscritos que atenderam a todos os critérios de inclusão. Os periódicos com maior número de publicações sobre o tema, cada um com sete artigos, foram: Revista de Saúde Pública, Arquivos Brasileiros de Cardiologia e Arquivos Brasileiros de Endocrinologia e Metabologia. A maior parte das publicações $(n=34)$ ocorreu nos últimos cinco anos (2003-2007), sendo que no período de 1993 a 2000 foram identificadas somente cinco publicações sobre o assunto.

Em 34 dos estudos revisados, a medida da relação cintura-quadril foi utilizada como indicador de obesidade centralizada, em 11 deles, a medida da circunferência da cintura e, em apenas três, o índice de conicidade. Este último indicador parece ter a sua aplicação bastante restrita, mesmo tendo sido proposto e apresentado na literatura especializada há mais de 16 anos. ${ }^{11}$ Somente um estudo adotou a relação circunferência da cintura-estatura como indicador da distribuição central da gordura corporal $^{12}$.

Na maioria dos estudos, a amostra foi muito heterogênea em relação à idade, sendo que dez incluíam adolescentes. Das investigações com amostras mais homogêneas em relação à faixa etária, quatro foram com idosos e três com crianças, mas nenhum com amostra exclusiva de adolescentes. Em 25 manuscritos, o tamanho da amostra foi superior a 500 sujeitos. Características dos artigos incluídos nesta revisão estão apresentadas na tabela 1.

Na tabela 2, apresenta-se um detalhamento de aspectos metodológicos de cada um dos estudos incluídos na revisão.

As amostras foram, geralmente, bastante heterogêneas, sendo que alguns estudos focalizaram a análise dessa inter-relação em subgrupos populacionais bastantes específicos, como em indígenas ${ }^{13}$, nipo-brasileiros ${ }^{24}$ e em população favelada ${ }^{46}$. Apenas um estudo foi conduzido exclusivamente com criança $^{50}$ e outro incluiu 14 crianças na amostra ${ }^{25}$, aspecto que sugere uma escassez de informações sobre a associação entre a obesidade abdominal e a ocorrência de hipertensão neste subgrupo.

Doze estudos ${ }^{2}, 13-15,24,25,27,28,46,49-51$ apresentaram evidências quanto à inter-relação entre obesidade centralizada e pressão arterial, na maior parte deles foram observadas correlações (ou associações) positivas, embora nem sempre estatisticamente significativas. Uma exceção foi o estudo de Sarni et $a 1^{50}$ que verificaram uma relação inversa $(r=-0,11)$, porém sem significância estatística, entre pressão arterial diastólica e circunferência da cintura em pré-escolares.

Foram observadas correlações significativas 
Tabela 1. Características gerais dos artigos incluídos na revisão.

\begin{tabular}{|c|c|c|}
\hline Característica & & Número de artigos \\
\hline Periódico & $\begin{array}{c}\text { Arquivos Brasileiros de Endocrinologia e Metabologia } \\
\text { Arquivos Brasileiros de Cardiologia } \\
\text { Jornal Brasileiro de Patologia e Medicina Laboratorial } \\
\text { Cadernos de Saúde Pública } \\
\text { Jornal Brasileiro de Pneumologia } \\
\text { Jornal de Pediatria } \\
\text { Revista Brasileira de Ginecologia e Obstetrícia } \\
\text { Revista da Associação Médica Brasileira } \\
\text { Revista Brasileira de Epidemiologia } \\
\text { Revista Brasileira de Medicina do Esporte } \\
\text { Revista de Nutrição } \\
\text { Revista de Saúde Pública } \\
\text { Revista Latino-Americana de Enfermagem }\end{array}$ & $\begin{array}{l}7 \\
7 \\
1 \\
6 \\
1 \\
1 \\
1 \\
5 \\
5 \\
1 \\
4 \\
7 \\
1\end{array}$ \\
\hline Período de publicação & $\begin{array}{c}\text { 1993-1996 } \\
\text { 1997-2000 } \\
\text { 2001-presente }\end{array}$ & $\begin{array}{c}1 \\
4 \\
42\end{array}$ \\
\hline Indicador antropométrico & $\begin{array}{c}\text { RCQ } \\
\text { IC } \\
\text { CC } \\
\text { RCEst }\end{array}$ & $\begin{array}{c}32 \\
3 \\
28 \\
1\end{array}$ \\
\hline Grupo estudado & $\begin{array}{c}\text { Crianças } \\
\text { Adultos ( } 20-60 \text { anos) } \\
\text { Idosos (>60 anos) } \\
\text { Heterogêneas }\end{array}$ & $\begin{array}{c}3 \\
13 \\
4 \\
27\end{array}$ \\
\hline Tamanho da amostra & $\begin{array}{c}<30 \\
31-99 \\
100-499 \\
\geq 500\end{array}$ & $\begin{array}{c}3 \\
10 \\
8 \\
26\end{array}$ \\
\hline
\end{tabular}

entre os indicadores e obesidade centralizada e os níveis de pressão arterial sistólica e diastólica em cinco $^{13,25,28,46,49}$ e três ${ }^{25,46,49}$ investigações, respectivamente. Entretanto, dois estudos ${ }^{14,51}$ evidenciaram que, após estratificação por sexo, as correlações entre indicadores antropométricos de obesidade centralizada e pressão arterial foram significativas somente entre os homens.

Verificou-se, ainda, uma prevalência de hipertensão em idosos que foi significativamente maior naqueles com RCQ superior a 0,96, independentemente do sexo². Entre os homens a prevalência foi de 63,7\% (enquanto entre sujeitos com RCQ<0,96 foi de $52 \%$ ), nas mulheres a proporção de hipertensas foi de $64,5 \%$ (enquanto entre aquelas com RCQ<0,96 foi de 38,7\%). Resultado similar foi observado por Souza e colaboradores ${ }^{27}$ que identificaram uma prevalência de hipertensão de 57,7\% em sujeitos com circunferência da cintura superior a 102 centímetros, enquanto entre os demais a proporção de hipertensos foi de $32 \%$.

Em estudo com nipo-brasileiros da cidade de São Paulo, Lerario et $a^{24}$ verificaram uma prevalência de obesidade abdominal de $67 \%$ e, além disso, que a ocorrência de hipertensão era $40 \%$ mais freqüente entre aqueles sujeitos que apresentaram RCQ elevada (0,95 para homens e
0,85 para mulheres). No mesmo estudo, os autores evidenciaram que a pressão arterial média foi significativamente maior no grupo que apresentou obesidade centralizada.

$O$ estudo de Gus et $\mathrm{a}^{15}$ evidenciou que sujeitos com RCQ inferior a 0,95 tendem a apresentar níveis de pressão arterial sistólica mais baixa. Os valores médios foram de $126 \mathrm{mmHg}$ (s=17) em sujeitos com RCQ inferior a 0,95 enquanto entre os demais este valor foi de $141 \mathrm{mmHg}(\mathrm{s}=25)$. Em relação aos níveis de pressão arterial diastólica não foram observadas diferenças significativas entre sujeitos com diferentes faixas de RCQ.

A análise da inter-relação entre obesidade abdominal e pressão arterial foi investigada também em pessoas diabéticas. Moraes et $\mathrm{al}^{25}$ verificaram uma correlação estatisticamente significativa entre circunferência da cintura e pressão arterial sistólica e diastólica, com coeficientes superiores a 0,5, que representam as magnitudes de correlação mais altas encontradas na presente revisão.

Foram localizados dez manuscritos $2,13,24-28,32,49,51$ que relataram evidências quanto a inter-relação de indicadores antropométricos de obesidade central com a ocorrência ou com parâmetros de controle clínico e metabólico do diabetes. Em quatro estudos, os autores relataram coeficientes de correlação 
Tabela 2. Características metodológicas dos estudos incluídos na revisão.

\begin{tabular}{|c|c|c|c|c|c|c|c|c|}
\hline \multirow[b]{2}{*}{ Autor(es) } & \multirow{2}{*}{ Ano } & \multicolumn{4}{|c|}{ Indicador Antropométrico } & \multirow{2}{*}{ Amostra } & \multirow{2}{*}{ Faixa Etária } & \multirow{2}{*}{ Localidade } \\
\hline & & RCQ & $\mathrm{CC}$ & IC & RCEst & & & \\
\hline Bloch et al ${ }^{13}$ & 1993 & $x$ & $x$ & & & 51 & $18-37$ & Surucucus, RO \\
\hline Guedes e Guedes ${ }^{14}$ & 1998 & $x$ & & & & 62 & $20-45$ & Londrina, PR \\
\hline Gus et $\mathrm{al}^{15}$ & 1998 & $x$ & $x$ & & & 1.088 & $18-61$ & Porto Alegre, RS \\
\hline Pereira et al ${ }^{16}$ & 1999 & $x$ & & & & 3.814 & $20-70$ & Rio de Janeiro, RJ \\
\hline Raskin et a $\left.\right|^{17}$ & 2000 & $x$ & & & & 518 & $45-65$ & Campinas, SP \\
\hline Cabrera e Jacob Filho² & 2001 & $x$ & & & & 1.847 & $60-94$ & Londrina, PR \\
\hline Navarro et $\mathrm{al}^{18}$ & 2001 & $x$ & & & & 157 & $35-75$ & Riberão Preto, SP \\
\hline Kac et $\mathrm{al}^{19}$ & 2001 & $x$ & $x$ & & & 781 & $16-45$ & Rio de Janeiro, RJ \\
\hline Velásquez-Melendez et $\mathrm{a}^{20}$ & 2002 & & $x$ & & & 791 & $15-59$ & Belo Horizonte, BH \\
\hline Afonso e Sichieri ${ }^{21}$ & 2002 & $x$ & & & & 1.446 & $20-60$ & Rio de Janeiro, RJ \\
\hline Cardoso et $\mathrm{al}^{22}$ & 2002 & $x$ & & & & 1.067 & $20-50$ & Cotia, SP \\
\hline Machado e Sichieri ${ }^{23}$ & 2002 & $x$ & & & & 2.441 & $20-60$ & Rio de Janeiro, RJ \\
\hline Lerario et $\mathrm{al}^{24}$ & 2002 & $x$ & & & & 530 & $40-79$ & São Paulo, SP \\
\hline Moraes et $\mathrm{al}^{25}$ & 2003 & & $x$ & & & 170 & $6-41$ & Rio de Janeiro, RJ \\
\hline Bahia e Gomes²6 & 2003 & $x$ & $x$ & & & 35 & $60-85$ & Rio de Janeiro, RJ \\
\hline Souza et $\mathrm{a}^{27}$ & 2003 & & $x$ & & & 1.039 & $18-70$ & Campos, RJ \\
\hline Carneiro et $\mathrm{al}^{28}$ & 2003 & $x$ & & & & 499 & $20-60$ & São Paulo, SP \\
\hline Martins e Marinho ${ }^{4}$ & 2003 & $x$ & $x$ & & & 1.047 & $20-60$ & São Paulo, SP \\
\hline Colombo et $\mathrm{al}^{29}$ & 2003 & $x$ & $x$ & & & 43 & $39-76$ & Campinas, SP \\
\hline Arruda et $\mathrm{al}^{30}$ & 2003 & $x$ & & & & 22 & $\geq 50$ & Amazônia Oriental, PA \\
\hline Pinto et $\mathrm{a}^{31}$ & 2003 & $x$ & & & & 71 & $31-77$ & Rio de Janeiro, RJ \\
\hline Andrade Júnior et $\mathrm{a}^{32}$ & 2004 & $x$ & $x$ & & & 64 & $27-36$ & Rio de Janeiro, RJ \\
\hline Cantos et $\mathrm{al}^{133}$ & 2004 & $x$ & & & & 52 & $18-60$ & Florianópolis, SC \\
\hline Soar et $\mathrm{al}^{34}$ & 2004 & $x$ & $x$ & & & 419 & $7-9$ & Florianópolis, SC \\
\hline Rasslan et a ${ }^{35}$ & 2004 & & $x$ & & & 96 & $20-45$ & São Paulo, SP \\
\hline Giugliano e Melo ${ }^{36}$ & 2004 & $x$ & & & & 528 & $6-10$ & Brasília, DF \\
\hline Pitanga e Lessa ${ }^{37}$ & 2004 & & & $x$ & & 968 & $30-74$ & Salvador, BA \\
\hline Taniguchi et al ${ }^{38}$ & 2004 & $x$ & $x$ & & & 647 & $35-60$ & São Paulo, SP \\
\hline Jaime et a ${ }^{39}$ & 2004 & & $x$ & & & 171 & $20-59$ & São Paulo, SP \\
\hline Da Cruz et al ${ }^{40}$ & 2004 & $x$ & & & & 196 & $80-86$ & Veranópolis, RS \\
\hline Scheffel et $\mathrm{al}^{41}$ & 2004 & $x$ & & & & 927 & $49-69$ & Porto Alegre, RS \\
\hline Monteiro et al ${ }^{42}$ & 2004 & $x$ & & & & 30 & $55-61$ & Amazonas, AM \\
\hline Florindo et al $\mathrm{l}^{43}$ & 2004 & $x$ & $x$ & & & 15 & $28-58$ & São Paulo, SP \\
\hline Pitanga e Lessa ${ }^{10}$ & 2005 & $x$ & $x$ & $x$ & & 968 & $30-74$ & Salvador, BA \\
\hline Cabrera et al ${ }^{44}$ & 2005 & $x$ & $x$ & & & 575 & $60-94$ & Londrina, PR \\
\hline Sampaio e Figueiredo 45 & 2005 & $x$ & $x$ & & & 643 & $20-72$ & Salvador, BA \\
\hline Ferreira et $\mathrm{al}^{46}$ & 2005 & $x$ & & & & 223 & $18-65$ & Maceió, AL \\
\hline Santos e Sichieri ${ }^{47}$ & 2005 & $x$ & $x$ & & & 2.005 & $40-60$ & Rio de Janeiro, RJ \\
\hline Barbosa et al ${ }^{48}$ & 2006 & & $x$ & & & 1.439 & adultos & Salvador, BA \\
\hline Rezende et al ${ }^{49}$ & 2006 & $x$ & $x$ & & & 231 & $21-76$ & Viçosa, MG \\
\hline Sarni et al ${ }^{50}$ & 2006 & & $x$ & & & 65 & $\begin{array}{l}\text { pré-esco- } \\
\text { lares }\end{array}$ & Santo André, SP \\
\hline Silva et al ${ }^{51}$ & 2006 & & $x$ & & & 99 & $20-65$ & Londrina, PR \\
\hline Castro et $a^{52}$ & 2006 & & $x$ & & & 753 & $\begin{array}{c}23 \text { anos } \\
(s=8)\end{array}$ & Rio de Janeiro, RJ \\
\hline Olinto et $\mathrm{al}^{53}$ & 2006 & & $x$ & & & 1.935 & $20-69$ & Pelotas, RS \\
\hline Pitanga e Lessa ${ }^{54}$ & 2006 & & & & $x$ & 968 & $30-74$ & Salvador, BA \\
\hline Freitas et al ${ }^{55}$ & 2007 & & $x$ & & & 768 & $>15$ & Ouro Preto, MG \\
\hline Pitanga e Lessa ${ }^{56}$ & 2007 & $x$ & $x$ & $x$ & & 968 & $30-74$ & Salvador, BA \\
\hline
\end{tabular}


entre indicadores de obesidade central e glicemia de jejum. Em três deles ${ }^{13,25,32}$ os coeficiente de correlação não foram estatisticamente significativos e a magnitude das correlações se situou em torno de 0,2. No estudo conduzido por Rezende e colaboradores ${ }^{49}$, o coeficiente de correlação entre as variáveis, apesar de baixa magnitude $(\mathrm{r}=0,24)$ foi estatisticamente significativo.

Apesar da aparente independência, a obesidade centralizada parece estar correlacionada a outros parâmetros de controle do diabetes. Moraes et a ${ }^{25}$ relataram uma correlação estatisticamente significativa, porém inversa $(\mathrm{r}=-0,31)$, entre a glicemia pós-prandial e a circunferência da cintura (denominada pelos autores como circunferência abdominal). A razão para o sentido inverso desta correlação não está estabelecida e futuros estudos deverão explorar melhor esta inter-relação.

Numa investigação com funcionários da EMBRAPA do Paraná, Silva et $a^{51}$ verificaram coeficientes de correlação estatisticamente significativos e de magnitude relativamente alta $(>0,5)$ entre a circunferência da cintura e o índice HOMA-IR de resistência à insulina. Após ajustamento pelo índice de massa corporal, a correlação entre estas variáveis foi significativa somente entre as mulheres $(\mathrm{r}=0,35)$.

Bahia e Gomes ${ }^{26}$, ao compararem diabéticos e não diabéticos verificaram não existir diferenças significativas entre os dois grupos quanto às medidas de circunferência da cintura e RCQ. Contrariamente, evidências apresentadas em quatro estudos ${ }^{2,24,27,28}$ sugerem que a prevalência de diabetes ou de tolerância anormal à glicose é maior em pessoas com obesidade centralizada. Lerario et $\mathrm{al}^{24}$, por exemplo, determinaram que a freqüência de diabetes em nipo-brasileiros foi $85 \%$ superior entre os sujeitos com RCQ elevada ( 0,85 para mulheres e 0,95 para homens). Resultados semelhantes foram verificados por Cabrera e Filho ${ }^{2}$ que, independente do sexo, identificaram maior proporção de diabéticos entre idosos com RCQ superior a 0,96.

Oito dos estudos revisados apresentaram evidências de inter-relação entre obesidade centralizada e parâmetros clínicos de controle das dislipidemias (colesterol total e frações, triglicérides) $\mathrm{e}$, somente dois ${ }^{4,50}$, relataram coeficientes de correlação ou medidas de associação que não atingiram significância estatística. Lerario et a ${ }^{124}$ observaram que a freqüência de dislipidemias foi cerca de 15\% superior entre sujeitos com RCQ elevada. Resultado similar ao observado por Souza et al. ${ }^{27}$ que identificaram, em estudo de base populacional realizado na Cidade de Campos (RJ), 32,2\% de casos de dislipidemias entre sujeitos com circunferência da cintura aumentada ( $>102$ centímetros) enquanto nos demais esta prevalência foi de $24,1 \%$.

Ainda sobre a prevalência de dislipidemias, vale destacar o trabalho desenvolvido por Cabrera e Jacob Filho ${ }^{2}$ que verificaram, em mulheres idosas, uma associação significativa entre indicadores de obesidade centralizada (RCQ e circunferência da cintura) e parâmetros clínicos de controle das dislipidemias (HDL-colesterol reduzido e triglicérides elevado). Barbosa e colaboradores ${ }^{48}$ observaram resultados semelhantes com adultos da Cidade de Salvador, exceto pelo fato de que, também entre os homens, a circunferência da cintura estava associada à ocorrência de hipertrigliceridemia.

Dois estudos ${ }^{49,51}$ analisaram a correlação entre a circunferência da cintura e níveis de triglicérides, colesterol total, LDL-colesterol e HDL-colesterol. Os resultados sugerem que, independente do sexo, a circunferência da cintura está positivamente correlacionada ao nível de triglicérides. Evidenciou-se, ainda, a correlação entre obesidade centralizada e HDL e LDL-colesterol, mas somente entre homens.

Sobre a correlação desses parâmetros clínicos de controle de dislipidemias com a RCQ, vale mencionar os resultados de dois estudos. Na investigação conduzida por Guedes e Guedes ${ }^{14}$, colesterol total e HDL-colesterol não apresentaram correlações significativas, entretanto, níveis de LDL e de triglicérides, correlacionaram-se significativamente com a RCQ, com coeficientes que variaram de 0,32 a 0,55. No estudo de Carneiro et $\mathrm{al}^{28}$, verificou-se entre os homens uma correlação significativa entre RCQ e todas as lipoproteínas avaliadas, enquanto nas mulheres isto foi observado somente em relação aos triglicérides.

Convém ainda discutir sobre os resultados dos dois únicos estudos que investigaram portadores de HIV/Aids e que foram publicados quase que simultaneamente. Num deles ${ }^{43}$, foram validados métodos para estimativa da gordura corporal, observando-se, mesmo após ajustamento pela idade, correlações estatisticamente significativas $(r \geq 0,80)$ entre circunferência da cintura e RCQ com medida efetuada por tomografia computadorizada. No estudo de Jaime e colaboradores ${ }^{39}$, procurou-se determinar a prevalência de obesidade abdominal com base na avaliação da medida da circunferência da cintura, verificando-se que $32,7 \%$ das mulheres e $6,4 \%$ dos homens apresentavam esta condição.

Três outros aspectos foram explorados nos estudos sobre obesidade centralizada e constituem inovações nas pesquisas realizadas nessa área e 
um campo ainda aberto para desenvolvimento de futuras investigações. Rasslan et $\mathrm{al}^{35}$ avaliaram a correlação entre indicadores de obesidade centralizada com parâmetros de função pulmonar, verificando que entre homens obesos a circunferência abdominal está significativa e positivamente correlacionada ao volume de reserva expiratório. A associação entre a adiposidade abdominal (RCQ) e a morbidade (mensurada por episódios de internação hospitalar) foi investigada por Afonso e Sichieri ${ }^{21}$. Os resultados evidenciaram que estes dois fatores estão fortemente associados $(\mathrm{OR}=3,5$; $\mathrm{p}=0,01)$. Por fim, vale destacar ainda os resultados do estudo de Cabrera e colaboradores ${ }^{44}$, que analisaram a relação entre obesidade abdominal (RCQ) e mortalidade, verificando que a deposição de gordura abdominal em mulheres idosas está associada à mortalidade por todas as causas, mas não está, neste grupo, relacionada à mortalidade por doenças cardiovasculares.

Faz-se necessário ainda comentar sobre a falta de padronização das medidas antropométricas utilizadas nos estudos. Apesar da disponibilidade de referências na literatura especializada, padronizando a realização destas medidas ${ }^{57,58}$, verificou-se grande variabilidade, particularmente, em relação à medida da circunferência da cintura (muitas vezes referidas inadequadamente pelos autores como circunferência abdominal). Este é um ponto a ser melhor considerado pois as diferenças e inconsistências de resultados entre os estudos podem ser devido a variações metodológicas adotadas pelos autores.

\section{CONSIDERAÇÕES FINAIS}

Intencionalmente, limitou-se esta revisão às publicações em periódicos indexados na SCIELO, aspecto que deverá ser considerado pelo leitor na análise dos resultados deste estudo. A despeito desta delimitação, os resultados sugerem que o corpo de conhecimento sobre o assunto sofreu grande ampliação, particularmente nos últimos cinco anos (2002-2007). Profissionais de saúde que utilizam a SCIELO como fonte de consulta e atualização científica não têm acesso a informações sobre a ocorrência de obesidade centralizada em adolescentes e noutros subgrupos populacionais ainda pouco investigados. Há também algumas linhas de investigação que podem ser mais exploradas, como o estudo da relação entre obesidade centralizada e mortalidade por causas não cardiovasculares.

\section{REFERÊNCIAS BIBLIOGRÁFICAS}

1. Popkin BM, Doak CM. The obesity epidemic is a worldwide phenomenon. Rev Nutr 1998; 56(4Pt 1):106-14.

2. Cabrera MAS, Jacob Filho W. Obesidade em idosos: prevalência, distribuição e associação com hábitos e co-morbidades. Arq Bras Endocrinol Metab 2001; 45(5):494-501.

3. World Health Organization. Food and Agriculture Organization [WHO/FAO]. Diet, nutrition and the prevention of chronic diseases. Geneva; 2003. [WHO - Technical Report Series, 916]

4. Martins IS, Marinho SP. O potencial diagnóstico dos indicadores da obesidade centralizada. Rev Saúde Pública 2003;37(6):760-767.

5. Welborn TA, Dhaliwal SS. Preferred clinical measures of central obesity for predicting mortality. Eur J Clin Nutr 2007;61(12):1373-1379.

6. Ghosh JR, Bandyopadhyay AR. Comparative evaluation of obesity measures: relationship with blood pressures and hypertension. Singap Med J 2007;48(3):232-235.

7. Hsieh SD, Yoshinaga H, Muto T. Waist-to-height ratio, a simple and practical index for assessing central fat distribution and metabolic risk in Japanese men and women. Int J Obes Relat Metab Disord 2003;27(5):610-616.

8. Hsieh SD, Muto T. The superiority of waist-to-height ratio as an anthropometric index to evaluate clustering of coronary risk factors among non-obese men and women. Prev Med 2005;40(2):216-220.

9. Molarius A, Seidell JC, Sans S, Tuomilehto J, Kuulasmaa K. Waist and hip circumferences, and waist-hip ratio in 19 populations of the WHO MONICA Project. Int J Obes Relat Metab Disord 1999;23(2):116-125.

10. Pitanga FJG, Lessa I. Anthropometric indexes of obesity as an instrument of screening for high coronary risk in adults in the city of Salvador - Bahia. Arq Bras Cardiol 2005;85(1):26-31.

11. Valdez R. A simple model-based index of abdominal adiposity. J Clin Epidemiol 1991;44(9):955-956.

12. Pitanga FJG, Lessa I. Razão cintura-estatura como discriminador do risco coronariano de adultos. Rev Assoc Med Bras 2006;52(3):157-161.

13. Bloch KV, Coutinho ESF, Lobo MSC, Oliveira JEP, Milech A. Blood pressure, capillary glucose, and anthropometric measurements in a Yanomámi population. Cad Saúde Pública 1993; 9(4):428-438.

14. Guedes DP, Guedes JERP. Distribuição de Gordura Corporal, Pressão Arterial e Níveis de Lipídios-Lipoproteínas Plasmáticas. Arq Bras Cardiol 1998;70(2):93-98.

15. Gus M, Moreira LB, Pimentel M, Gleisener ALM, Moraes RS, Fuchs FD. The association between different measurements of obesity and the prevalence of hypertension. Arq Bras Cardiol 1998;70(2):111-114.

16. Pereira RA, Sichieri R, Marins VMR. Razão cintura/ quadril como preditor de hipertensão arterial. Cad Saúde Pública 1999;15(2):333-344.

17. Raskin DBF, Pinto-Neto AM, Paiva LHSC, Raskin A, Martinez EZ. Fatores Associados à Obesidade e ao Padrão Andróide de Distribuição da Gordura Corporal em Mulheres Climatéricas. Rev Bras Ginecol Obstet 2000;22(7):435-441. 
18. Navarro AM, Stedille MS, Unamuno MRDL, Marchini JS. Body fat distribution in patients with and without chronic-degenerative diseases: use of the waist to hip relationship and arm fat index. Rev Nutr 2001;14(1):37-41.

19. Kac G, Velásquez-Meléndez G, Coelho MASC. Fatores associados à obesidade abdominal em mulheres em idade reprodutiva. Rev Saúde Pública 2001;35(1):46-51.

20. Velasquez-Melendez G, Kac G, Valente JG, Tavares R, Silva CQ, Garcia ES. Evaluation of waist circumference to predict general obesity and arterial hypertension in women in Greater Metropolitan Belo Horizonte, Brazil. Cad Saúde Pública. 2002;18(3):765-771.

21. Afonso FM, Sichieri R. Associação do índice de massa corporal e da relação cintura/quadril com hospitalizaÇões em adultos do Município do Rio de Janeiro, RJ. Rev Bras Epidemiol 2002;5(2):153-63.

22. Cardoso E, Martins IS, Fornari L, Monachini MC, Mansur AP, Caramelli B. Electrocardiographic abnormalities and cardiovascular risk factors for ischemic heart disease in an adult population from São Paulo, Brazil. Rev Assoc Med Bras (1992). 2002;48(3):231-236.

23. Machado PAN, Sichieri R. Relação cintura-quadril e fatores de dieta em adultos. Rev Saúde Pública 2002;36(2):198-204.

24. Lerario DDG, Gimeno SG, Franco LJ, Iunes M, Ferreira SRG. Excesso de peso e gordura abdominal para a síndrome metabólica em nipo-brasileiros. Rev Saúde Pública 2002;36(1):4-11.

25. Moraes CM, Portella RB, Pinheiro VS, Oliveira MMS, Fuks AG, Cunha EF et al. Prevalence of overweight and obesity in type 1 diabetic patients. Arq. Bras Endocrinol Metab 2003;47(6):677-683.

26. Bahia L, Gomes MB. Influência da idade e do diabetes sobre esteróides sexuais e SHBG em homens. Arq Bras Endocrinol Metab 2003;47(3):256-260.

27. Souza LJ, Gicovate Neto C, Chalita FEB, Reis AFF, Bastos DA, Souto Filho JTD et al. Prevalência de obesidade e fatores de risco cardiovascular em Campos, Rio de Janeiro. Arq Bras Endocrinol Metab 2003;47(6):669-676.

28. Carneiro G, Faria AN, Ribeiro Filho FF, Guimarães A, Lerario D, Ferreira SRG et al. Influência da distribuição da gordura corporal sobre a prevalência de hipertensão arterial e outros fatores de risco cardiovascular em indivíduos obesos. Rev Assoc Med Brás 2003;49(3):306-311.

29. Colombo RCR, Aguilar OM, Gallani MCBJ, Gobatto CA. Caracterização da Obesidade em pacientes com infarto do miocárdio. Rev Latino-Am Enfermagem 2003;11(4):461-467.

30. Arruda HO, Vieira Filho JPB, Ortiz V, Srougi M. PSA e medidas antropométricas em índios da Amazônia: avaliação da comunidade Parkatejê. Rev Saúde pública 2003;37(5):624-628.

31. Pinto VLM, Meirelles LR, Farinatti PTV. Influência de programas não-formais de exercícios (doméstico e comunitário) sobre a aptidão física, pressão arterial e variáveis bioquímicas em pacientes hipertensos. Rev Bras Med Esporte 2003;9(5):267-274.

32. Andrade JR, Carlos RM, Clemente EL, Gomes MB. Body adiposity and its influence on clinical and meta- bolic parameters of patients with type 1 diabetes. Arq Bras Endocrinol Metab 2004;48(6):885-889.

33. Cantos GA, Duarte MFS, Dutra RL, Silva CSM, Waltrick CDA, Balen MG et al. Prevalence of risk factors of coronary artery disease in university hospital employees and the correlation with psychological stress. J Bras Patol Med Lab 2004; 40(4):240-247.

34. Soar C, Vasconcelos FAG, Assis MAA. A relação cintura quadril e o perímetro da cintura associados ao índice de massa corporal em estudo com escolares. Cad Saúde Pública 2004;20(6):1609-1616

35. Rasslan Z, Saad Junior R, Stirbulov R, Fabbri RMA, Lima CAC. Avaliação da função pulmonar na obesidade graus I e II. J Bras Pneumol 2004;30(6):508-514.

36. Giugliano R, Melo ALP. Diagnosis of overweight and obesity in schoolchildren: utilization of the body mass index international standard. J Pediatr (Rio J.) 2004; 80(2):129-134.

37. Pitanga FJG, Lessa I. Sensibilidade e especificidade do índice de conicidade como discriminador do risco coronariano de adultos em Salvador, Brasil. Rev Bras Epidemiol 2004;7(3):259-269.

38. Taniguchi C, Gimeno SGA, Ferreira SRG, JapaneseBrazilian Diabetes Study Group. Anthropometric characteristics of Japanese-Brazilians. Rev Bras Epidemiol 2004;7(4):423-433.

39. Jaime PC, Florindo AA, Latorre MRDO, Brasil BG, Santos ECM, Segurado AAC. Prevalence of overweight and central obesity in HIV/AIDS patients treated with highly active antiretroviral therapy. Rev Bras Epidemiol 2004;7(1):65-72.

40. Da Cruz IBM, Almeida MSC, Schwanke CHA, Moriguchi EH. Obesity prevalence among oldest-old and its association with risk factors and cardiovascular morbidity. Rev Assoc Med Bras (1992) 2004;50(2):172-177.

41. Scheffel RS, Bortolanza D, Weber CS, Costa LA, Canani LH, Santos KG et al. Prevalência de complicações micro e macrovasculares e de seus fatores de risco em pacientes com diabetes melito do tipo 2 em atendimento ambulatorial. Rev Assoc Med Bras 2004;50(3):263-267.

42. Monteiro RCA, Riether PTA, Burini RC. The effects of a mixed program of nutritional intervention and physical exercise on body composition and feeding habits of obese climacteric women. Rev Nutr 2004;17(4):479-489.

43. Florindo AA, Latorre MRDO, Santos ECM, Borelli A, Rocha MS, Segurado AAC. Validation of methods for estimating HIV/AIDS patients' body fat. Rev. Saúde Pública. 2004;38(5):643-649.

44. Cabrera MAS, Wajngarten M, Gebara OCE, Diament J. Relação do índice de massa corporal, da relação cintura-quadril e da circunferência abdominal com a mortalidade em mulheres idosas: seguimento de 5 anos. Cad Saúde Pública 2005; 21(3):767-775.

45. Sampaio LR, Figueiredo VC. Correlação entre o índice de massa corporal e indicadores antropométricos de distribuição de gordura corporal em adultos e idosos. Rev Nutr 2005;18(1):53-61.

46. Ferreira HS, Florêncio TMTM, Fragoso MAC, Melo FP, Silva TG. Hypertension, abdominal obesity and 
short stature: aspects of nutritional transition within a shantytown in the city of Maceió (Northeastern Brazil). Rev Nutr 2005;18(2):209-218.

47. Santos DM, Sichieri R. Body mass index and measures of adiposity among elderly adults. Rev Saúde Pública 2005;39(2):163-168.

48. Barbosa PJB, Lessa O, Filho NA, Magalhães LBNC, Jenny A. Critério de obesidade central em população brasileira: impacto sobre a síndrome metabólica. Arq Bras Cardiol 2006;87:407-414.

49. Rezende FAC, Rosado LEFPL, Riberio RCL, Vidigal FC, Vasques ACJ, Bonard IS et al. Índice de massa corporal e circunferência abdominal: associação com fatores de risco cardiovascular. Arq Bras Cardiol 2006;87(6):728-734.

50. Sarni RS, Souza FIS, Schoeps DO, Catherino P, Oliveira MCCP, Pessotti CFX et al. Relação da cintura abdominal com a condição nutricional, perfil lipídico e pressão arterial em pré-escolares de baixo estrato socioeconômico. Arq Bras Cardiol 2006;87(2):153-158.

51. Silva JLT, Barbosa DS, Oliveira JA, Guedes DP. Distribuição centrípeta da gordura corporal, sobrepeso e aptidão cardiorrespiratória: associação com sensibilidade insulínica e alterações metabólicas. Arq Bras Endocrinol Metab 2006;50(6):1034-1040.

52. Castro SH, Mato HJ, Gomes MB. Parâmetros antropométricos e síndrome metabólica em diabetes tipo 2. Arq Bras Endocrinol Metab 2006;50(3):450-455.

53. Olinto MTA, Nácul LC, Dias-da-Costa JS, Gigante DP, Menezes AMB, Macedo S. Níveis de intervenção para obesidade abdominal: prevalência e fatores associados. Cad Saúde Pública 2006;22(6):1207-1215.
54. Pitanga FJG, Lessa I. Razão cintura-estatura como discriminador do risco coronariano de adultos. Rev Assoc Med Bras 2006;52(3):157-161.

55. Freitas SN, Caiaffa WT, César CC, Faria VA, Nascimento RM, Coelho GLLM. Risco nutricional na população urbana de Ouro Preto, sudeste do Brasil: estudo de corações de Ouro Preto. Arq Bras Cardiol 2007;88(2):191-199.

56. Pitanga FJG; Lessa I. Associação entre indicadores antropométricos de obesidade e risco coronariano em adultos na cidade de Salvador, Bahia, Brasil. Rev Bras Epidemiol 2007;10(2):239-48.

57. Petroski EL. Antropometria Técnicas e Padronizações. Santa Maria: Pallotti; 2003.

58. Lohman TG, Roche AF, Martorell R. Anthropometric standardization reference manual. Champaign: Human Kinetics Books; 1991.

\section{Endereço para correspondência}

Cláudio Barnabé dos Santos Cavalcanti

Rua Arnóbio Marques, 310, Campus Universitário HUOC - Santo Amaro

CEP: 50100-130 - Santo Amaro, Recife, PE.

E-mail: cbarnabe@terra.com.br 